\title{
Auswirkungen einer Liberalisierung im Agrarsektor auf Entwicklungsländer und die Schweiz
}

Les conséquences d'une libéralisation du secteur agricole pour les pays en développement et la Suisse

Heidi Bravo-Baumann

\section{OpenEdition}

\section{Journals}

\section{Electronic version}

URL: http://journals.openedition.org/sjep/1242

DOI: $10.4000 /$ sjep.1242

ISSN: 1663-9677

Publisher

Institut de hautes études internationales et du développement

\section{Printed version}

Date of publication: 1 février 1993

Number of pages: 173-191

ISSN: 1660-5926

\section{Electronic reference}

Heidi Bravo-Baumann, « Auswirkungen einer Liberalisierung im Agrarsektor auf Entwicklungsländer und die Schweiz », Schweizerisches Jahrbuch für Entwicklungspolitik [Online], 12 | 1993, Online erschienen am: 30 April 2013, abgerufen am 08 September 2020. URL : http:// journals.openedition.org/sjep/1242; DOI : https://doi.org/10.4000/sjep.1242 


\title{
Auswirkungen einer Liberalisierung im Agrarsektor auf Entwicklungsländer und die Schweiz*
}

\author{
Heidi Bravo-Baumann
}

\section{Einleitung}

Nach einer Phase der relativen Stabilität in den 60er Jahren sind die internationalen Agrarmärkte zunehmend durch Überschüsse und grosse Preissprünge gekennzeichnet. Für Agrarexportländer ist es immer schwieriger geworden, mit einigermassen stabilen Einkommen aus dem Agrarhandel zu rechnen. Gleichzeitig haben die westlichen Industrieländer zunehmend protektionistische Massnahmen zum Schutz ihres Agrarsektors getroffen. Dies führte zu steigenden Überschüssen, welche nur dank Exportsubventionen abgesetzt werden konnten und die Weltmarktpreise negativ beeinflussten. Die agrarpolitischen Massnahmen zugunsten der eigenen Produzenten und die Verwertungskosten haben mittlerweile ein Ausmass angenommen, welches das Budget der Industrieländer stark belastet.

In der Uruguay-Runde der GATT-Verhandlungen wurde die Agrarfrage erstmals aufgenommen, doch konnte bis heute noch keine Einigung erzielt werden. Das Ziel dieses Artikels besteht darin, Perspektiven des Weltagrarhandels unter den zu erwartenden Änderungen der Liberalisierung abzuschätzen. Ausgehend von der heutigen Situation soll aufgezeigt werden, welche Auswirkungen eine Liberalisierung des Agrarhandels auf Entwicklungsländer einerseits und die Schweiz anderseits hätte. In einem weitern Schritt soll die Frage aufgenommen werden, wie sich der Agrarhandel bei einer Liberalisierung zwischen der Schweiz und Ländern der Dritten Welt verändern würde.

* Cf. résumé p. 261. 


\section{Situation auf den Agrarweltmärkten}

\subsection{Entwicklungen auf den Agrarweltmärkten}

Der Welthandel mit Agrarprodukten hat erst in den letzten hundertfünfzig Jahren eine grosse Ausdehnung erfahren, infolge des Fortschritts im Transportwesen und in der Lagerhaltung. Während sich der Agrarwelthandel in den Anfängen auf dauerhafte Produkte (z.B. Gewürze, Farbstoffe, Textilfasern) beschränkte, sind heute quasi alle landwirtschaftlichen Erzeugnisse handelbar. Selbst verderbliche Produkte wie tropische Früchte können mittels Flugtransport innert kürzester Frist aus der Dritten Welt auf die Märkte der Industrieländer gebracht werden.

Seit dem letzten Weltkrieg hat das Volumen des internationalen Agrarhandels stetig zugenommen, allein zwischen 1963 und 1983 erfolgte ein Anstieg um rund $90 \%$. Gleichzeitig ist aber das gesamte Welthandelsvolumen um rund $180 \%$ gestiegen, so dass der Anteil des Weltagrarhandels am gesamten Welthandel in dieser Zeitspanne von rund $27 \%$ auf $15 \%$ gesunken ist (Egger, Rieder, Clemenz, 1992). Wichtigste Akteure des internationalen Agrarhandels sind die Industrieländer. Fast die Hälfte des Handels wickelt sich innerhalb dieser Gruppe ab. Ein weiteres Drittel des Agrarhandels erfolgt zwischen Industrie- und Entwicklungsländern und rund $10 \%$ zwischen Entwicklungsländern.

Wie Tabelle 1 zeigt, haben die Länder der Dritten Welt in den letzten 30 Jahren ständig Marktanteile an die Industrieländer verloren. Dies gilt besonders für den Bereich der Nahrungsmittel. Im internationalen Handel mit Getränken (Tee, Kaffee, Kakao) und Rohstoffen bleiben die Entwicklungsländer Hauptexporteure mit 93\% bzw. 65\% der Gesamtexporte (Egger, Rieder, Clemenz, 1992).

\subsection{Instabilitäten auf den internationalen Agrarmärkten}

In den letzten Jahrzehnten ist das Angebot auf den Weltagrarmärkten stärker als die Nachfrage angestiegen, was tendenziell zu sinkenden Preisen führte (siehe Abbildung 1). Ein weiteres wichtiges Merkmal der Weltagrarmärkte ist die Instabilität der Preise. Trotz moderner Produktionsmethoden wird das Angebot durch witterungsbedingte Ernteschwankungen geprägt. So verknappen beispielsweise durch Frost bedingte Ertragsausfälle bei mehrjährigen Pflanzen wie z.B. Kaffee das Angebot für einige Jahre, da es mehrere Jahre dauert bis Neupflanzungen den vollen Ertrag abwerfen. Die Weltmarktpreise werden infolge dieser Verknappung ansteigen und die Produzenten werden auf diesen Preisanstieg mit vermehrten Investitionen reagieren. Kommen nach einigen Jahren die Erträge der Neupflanzungen zusätzlich auf den Markt, werden zwangsläufig Überschüsse entstehen und die Preise zusammenbrechen. Das Angebot ist somit kurzfristig starr oder inelastisch. Bei Mangel kann nicht sofort mehr produziert werden, wāhrend bei Überschusslagen jeder Produzent auf Einnahmen angewiesen ist und daher sein Angebot kurzfristig kaum wesentlich verkleinert. 
Tabelle 1: Exportanteile verschiedener Ländergruppen bei Agrarprodukten

\begin{tabular}{|l|c|c|c|c|}
\hline & Jahr & $\begin{array}{c}\text { Industrie- } \\
\text { länder }\end{array}$ & Dritte Welt & $\begin{array}{c}\text { Osteuropa } \\
\text { und UdSSR }\end{array}$ \\
\hline Getränke & $1961-63$ & 1.7 & 98.1 & 0.2 \\
& $1982-84$ & 4.7 & 94.9 & 0.4 \\
& $1988-89$ & 46.2 & 44.8 & 8.9 \\
\hline Nahrungsmittel & $1961-63$ & 46.2 & 44.8 & 8.9 \\
& $1982-84$ & 62.7 & 34.2 & 3.0 \\
\hline Rohstoffe & $1988-89$ & 77.6 & 18.1 & 4.3 \\
\hline Agrarprodukte & $1961-63$ & 23.5 & 69.2 & 7.3 \\
total & $1982-84$ & 24.0 & 65.3 & 10.7 \\
& $1988-89$ & 26.4 & 64.6 & 9.0 \\
\hline & $1961-63$ & 30.5 & 63.1 & 6.4 \\
\hline & $1982-84$ & 47.9 & 48.4 & 3.6 \\
\hline
\end{tabular}

Anmerkung: Exportmengen wurden mit Durchschnittspreisen der Weltexporte 197882 gewichtet. Getränke umfassen Kaffee, Kakao, Tee; Nahrungsmittel sind Getreide, Zucker, Fleisch, Milchprodukte, Wurzel- und Knollengewächse, Hülsenfrüchte, Obst und Gemüse; Rohstoffe bestehen aus Baumwolle, Jute, Kautschuk und Tabak. Datenbasis: FAO, Trade Yearbooks

Quelle: Egger, Rieder, Clemenz, 1992

Mittels internationaler Abkommen wurde versucht, die Märkte gewisser Produkte wie Kaffee, Kakao und Zucker zu stabilisieren. Die Praxis hat allerdings gezeigt, dass trotz internationaler Abkommen die Märkte nicht stabilisiert und der Preiszerfall nicht aufgehalten werden konnte.

Die Abkommen sind meist daran gescheitert, dass einerseits die Vorstellungen der Industrie- und Entwicklungsländer nicht in Einklang gebracht werden konnten und andererseits die Quotenregelungen von den Exporteuren nicht eingehalten wurden. In Zeiten hoher Agrarpreise, wenn die Weltmarktpreise sich am oberen Rand des vereinbarten Preisbands bewegen, ist das Interesse der Exporteure an stabilen Preisen geringer. Sinken die Preise infolge von Überschüssen, ist die Einhaltung der Quoten nicht gewährleistet. Einzelne Produzentenländer werden in diesem Fall versuchen, die Devisenausfälle mit höheren Ausfuhren wettzumachen, wodurch der Weltmarktpreis noch mehr unter Druck gerät. 


\section{Abbildung 1: Entwicklung der Nahrungsmittelpreise}

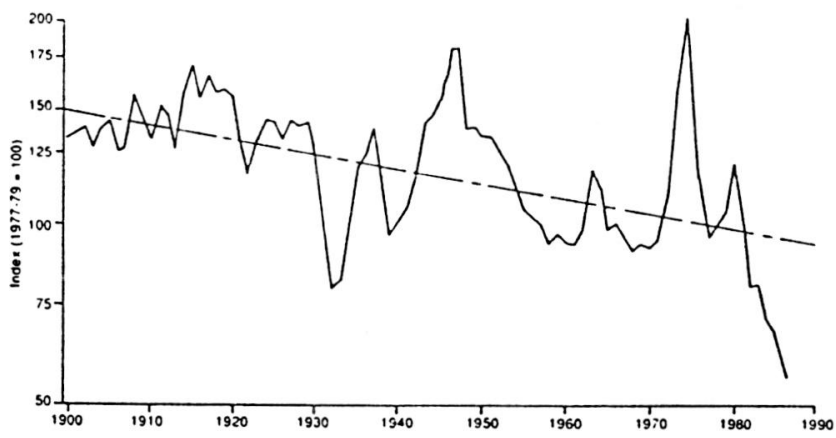

Quelle: Egger, Rieder, Clemenz, 1992

\subsection{Partner im internationalen Weltagrarhandel}

Wie Abbildung 2 zeigt, gibt es auf dem Weltagrarhandel dominante und abhängige Partner. Die EG und die USA bestimmen mit ihrer Politik der Exportsubventionierung die Lage auf dem Weltmarkt. Das Abschöpfungssystem der EG führte zu relativ stabilen Preisen im Innern, was Angebotsausdehnungen bewirkte und in der Folge zunehmende Überschüsse. Um diese Überschüsse auf den Weltmärkten abzusetzen, müssen Exportsubventionen ausgerichtet werden. Dadurch verloren die USA als traditioneller Agrarexporteur den europäischen Markt und ergriffen daher ebenfalls Massnahmen zur Stützung ihrer Landwirte. Als Folge stiegen die Ausgaben zur Stützung der Landwirtschaft und die Kosten der Überschussverwertung stetig. Unter den heutigen Budgetrestriktionen sind die Industrieländer gezwungen, auch die Ausgaben für die Landwirtschaft zu redimensionieren. Neben diesen Budgetproblemen haben die unregelmässig anfallenden Überschüsse aber auch zur Erhöhung der Preisinstabilitäten auf den Weltmärkten beigetragen.

Durch die erwähnten agrarpolitischen Massnahmen wurde den Ländern der Dritten Welt der Zugang zu den Agrarmärkten für Nahrungsmittel zunehmend verwehrt. Die Marktanteile traditioneller Agrarexporteure wie Argentinien sind geschwunden. Andere Entwicklungsländer wurden infolge des Bevölkerungswachstums und/oder geringer Anreize für die Inlandproduktion zu Nahrungsmittelimporteuren. Tiefe Weltmarktpreise beeinflussen die Politik der Entwicklungsländer. Um die Versorgung der Grossstädte sicherzustellen, importieren die meisten Regierungen der Entwicklungsländer preisgünstige Nahrungsmittel, wovon die Konsumenten profitieren. Die Landwirtschaft hingegen verliert wichtige Produktionsanreize, wenn die Produktion für den Inlandmarkt in diesen Ländern nicht geschützt wird und sich das tiefe Weltmarktpreisniveau ins Innere der Länder 
überträgt. Die Folge sind eine Produktionsabnahme und die Erhöhung der Abhängigkeit von Importen.

Von den günstigen Weltmarktpreisen für Agrargüter profitieren vor allem die importierenden kleinen Industrieländer. So stehen der Schweiz einerseits stets beliebige Importmengen zu tiefen Preisen zur Vertügung. Anderseits müssen landwirtschaftliche Exporte durch Subventionen verbilligt werden, damit die Produkte abgesetzt werden können. Zur Finanzierung eines beachtlichen Teils des schweizerischen Agrarschutzes werden Einfuhrabgaben erhoben, wodurch sich die Konsumentenpreise erhöhen.

\section{Abbildung 2: Akteure und Partner im Weltagrarhandel}

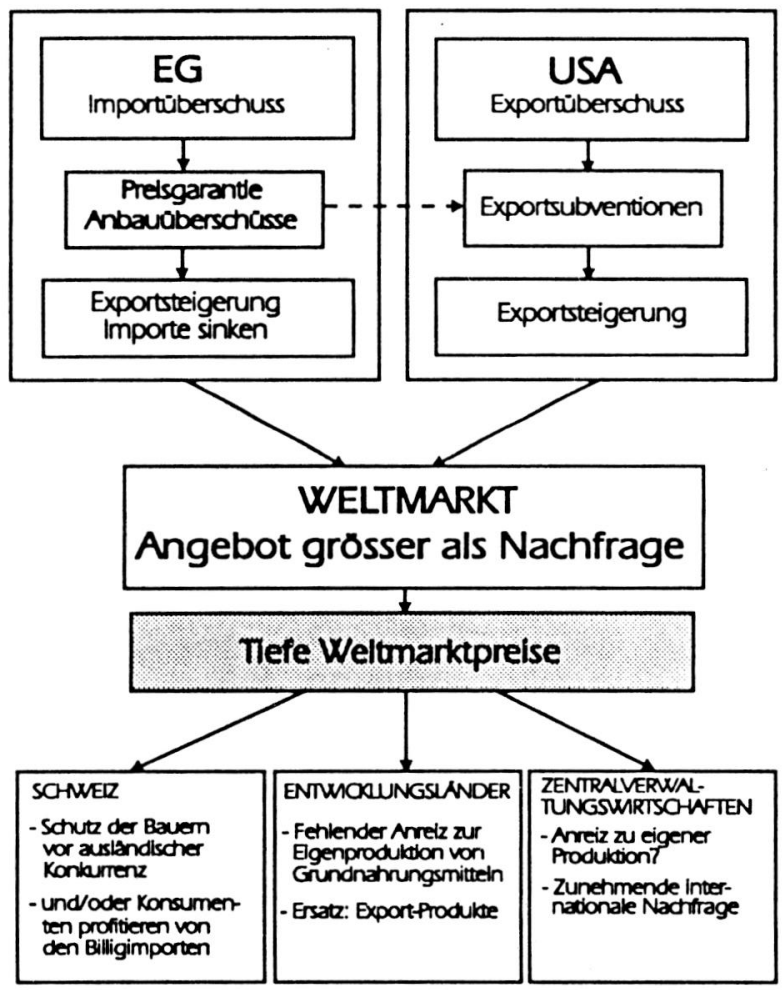




\section{Aussichten einer Liberalisierung des Agrarhandels}

Die bisherigen Ausführungen haben deutlich gemacht, dass die Weltagrarmärkte seit dem zweiten Weltkrieg zunehmend instabiler geworden sind. Trotz Überschüssen im Norden haben sich die Armuts- und Unterernährungsprobleme im Süden absolut betrachtet weiter verstärkt. Eine ähnliche Entwicklung wie im Süden ist für Osteuropa zumindest nicht unwahrscheinlich.

Das Ziel des 1948 gegründeten Abkommens General Agreement on Tariffs and Trade (GATT) ist die schrittweise Liberalisierung des Welthandels. Die verschiedenen Verhandlungsrunden im Rahmen der GATT seit den 60er Jahren bewirkten einen durchschnittlichen Abbau der Zölle bei Industrieprodukten von rund $40 \%$ auf etwa 5\% (Egger, Rieder, Clemenz, 1992). Beim Agrarhandel ist gerade die umgekehrte Tendenz festzustellen, nämlich eine Zunahme der Zölle auf rund $40 \%$. Dieser Gegensatz zwischen der verstärkten Integration der Weltwirtschaft bei gleichzeitig zunehmender Abschottung der nationalen Agrarmärkte ist eines der zentralen Spannungsfelder im Welthandel.

Die gesamthaft unbefriedigende Situation auf dem Agrarweltmarkt führte im Rahmen der Uruguay-Runde (Beginn 1986) zur erstmaligen Aufnahme der Agrarfrage in die GATT-Verhandlungen. Ziel dieser Verhandlungen ist es, die Verzerrungen des internationalen Agrarhandels in den einzelnen Ländern abzubauen und auch hier den freien Handel zu fördern. Dies bedeutet den Abbau von Importbarrieren, die Ausarbeitung genauer Bestimmungen über zulässige direkte und indirekte Subventionen an die Landwirtschaft, den Abbau nichttarifärer Handelshemmnisse (z.B. sanitäre und phytosanitäre Bestimmungen), welche den Agrarhandel beeinträchtigen. Es ist allerdings, wie die immer noch laufenden Verhandlungen zeigen, nicht einfach, die divergierenden Interessen und Prioritäten der einzelnen Länder(gruppen) auf einen Nenner zu bringen.

So konnte man sich in den Schlussverhandlungen im Dezember 1990 in Brūssel erwartungsgemäss nicht auf einen einheitlichen Abbau der Agrarsubventionen einigen. Während die USA und die CAIRNS-Gruppe einen Abbau der Stützungsmassnahmen von rund $70 \%$ verlangten, blieb die $E G$ bei ihrer Offerte einer Kürzung um 30\%. Das Angebot der Schweiz in dieser Runde sah eine Reduktion der inneren Stützung von $20 \%$ und eine Reduktion der Budgetausgaben für Exportsubventionen von 30\% vor. Im Januar 1991 wurden die Verhandlungen in Genf wieder aufgenommen, doch ist eine Einigung zum heutigen Zeitpunkt (Oktober 1992) noch nicht in Sicht.

\section{Mögliche Auswirkungen einer Liberalisierung im Agrarhandel auf die Schweiz}

\subsection{Problemkreise der schweizerischen Landwirtschaft}

Im Falle einer Liberalisierung im Agrarhandel ist für die Schweiz absehbar, dass das heutige starre System des Aussenhandelsschutzes und der Mengenbe- 
schränkung gelockert werden muss. Die GATT-Verhandlungen sind aber nur eine der Herausforderungen, welchen sich die schweizerische Landwirtschaft stellen muss. Gleichzeitig stehen ein möglicher Beitritt zum EWR und zu einem späteren Zeitpunkt eventuell gar zurEG zur Diskussion. Die EWR-Verhandlungen haben auf die Agrarpolitik der Schweiz einen geringen Einfluss, da dieser Sektor vom Vertrag ausgenommen ist. Die Einflüsse wären allenfalls indirekt über den Arbeitsmarkt und durch die Liberalisierung im Produktionsmittelhandel spürbar. Durch den aussenpolitischen Druck können aber heute die seit geraumer Zeit bestehenden Problemkreise der schweizerischen Landwirtschaft unbefangener angegangen werden. Wie Abbildung 3 zeigt, stellen sich der schweizerischen Landwirtschaft vier Grundprobleme: das Mengen- und Überschussproblem, das Problem der Ökologie, der Einkommenssicherung und das Strukturproblem.

\section{a) Das Mengen- und Überschussproblem}

Im Zentrum der seit vierzig Jahren betriebenen schweizerischen Agrarpolitik steht der im Landwirtschaftsgesetz verankerte Grundsatz der kostendeckenden Preise mit dem Ziel, das bäuerliche Einkommen über die Preise zu sichern. Gemäss Artikel 23 des Landwirtschaftsgesetzes kann die Einfuhr landwirtschaftlicher Produkte eingeschränkt werden, sofern der Absatz der Inlandproduktion zu kostendeckenden Preisen durch die Einfuhren gefährdet ist.

Wie aus Tabelle 2 ersichtlich wird, werden in der Schweiz zur Lenkung der Einfuhr landwirtschaftlicher Produkte unterschiedliche Instrumente angewandt. So sind zum Beispiel die Importe von Frischmilch und kurantem Weisswein verboten, während diejenigen von Futtergetreide, Fleisch und Fasswein mengenmässig beschränkt sind. Die Einfuhr anderer Produkte wie Brotgetreide und Eier wiederum ist an die Übernahme der Inlandprodukte gebunden (Leistungssystem). Obst und Gemüse sind der zeitlichen Mengenbeschränkung unterstellt (Dreiphasensystem), das heisst ihre Einfuhr wird zuerst beschränkt und dann verboten, sobald die Inlandangebote auf den Markt gelangen. Zusätzlich werden auf die meisten importierten Produkte Zoll- oder Preiszuschläge erhoben.

Generell sind die Einfuhrbestimmungen vom Selbstversorgungsgrad abhängig; je höher der Selbstversorgungsgrad, desto mehr muss der Inlandmarkt geschützt werden und umso restriktiver sind die Importbestimmungen. Die Weltmarktpreise für entsprechende Produktqualitäten liegen meist bedeutend unter den schweizerischen Inlandpreisen, da die Faktorkosten im Inland verhältnismässig hoch sind.

\section{b) Das Ökologieproblem}

Die Intensivierung der Produktion hat zu zunehmender Belastung der Umwelt auch durch die Landwirtschaft geführt. Durch die reale Verbilligung der Inputs und den Anstieg der Produzentenpreise wurde eine Erhöhung des Einsatzes landwirtschaftlicher Inputs lohnend. Jedermann stimmt heute der Forderung zu, dass die Landwirtschaft keine nachhaltigen Umweltschäden verursachen und Nahrungs- 


\section{Abbildung 3: Problemkreise der schweizerischen Landwirtschaft}

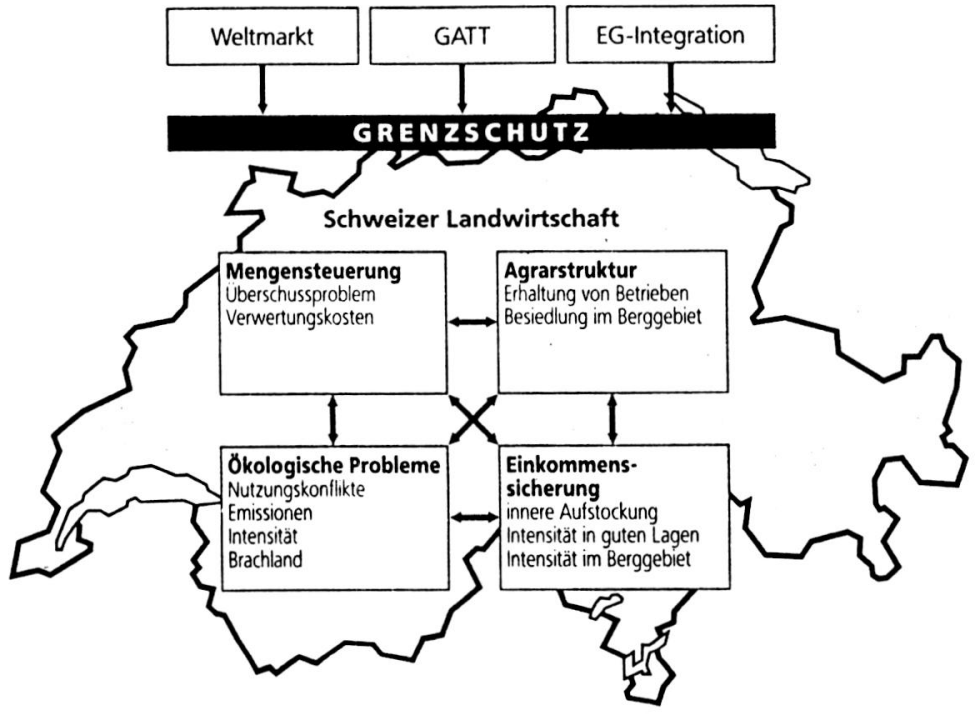

Quelle: Rieder, 1991

mittel ohne chemische Rückstände produzieren soll. Ökologische Schäden können durch Gesetze (z.B. Gewässerschutzgesetz) verhindert werden. Auch ist bekannt, dass grössere Betriebe aus wirtschaftlichen Gründen extensiver produzieren können als Kleinbetriebe. Die Betriebe werden deshalb versuchen, Einkommenseinbussen - verursacht durch ökologische Auflagen und damit Extensivierungszwang - durch Betriebsvergrösserungen aufzufangen.

\section{c) Das Problem der Einkommenssicherung}

Heute beruhen die Einkommen der Bauern auf zwei Pfeilern: dem Einkommen aus der Produktion und den Direktzahlungen. Dem Einkommen aus der Produktion sind heute durch die quantitativen Restriktionen enge Grenzen gesetzt. Bauern betrachten sich als Unternehmer und sträuben sich gegen eine klare Trennung zwischen Einkommen und produktionsunabhängigen Direktzahlungen. Durch die quantitativen Einschränkungen sind aber einerseits dem freien Unternehmertum enge Grenzen gesetzt, anderseits wird dem Bauern auch heute über Direktzah- 


\section{Tabelle 2: Aussenhandelsbeschränkungen der Schweiz}

Die wichtigsten Märkte mit:

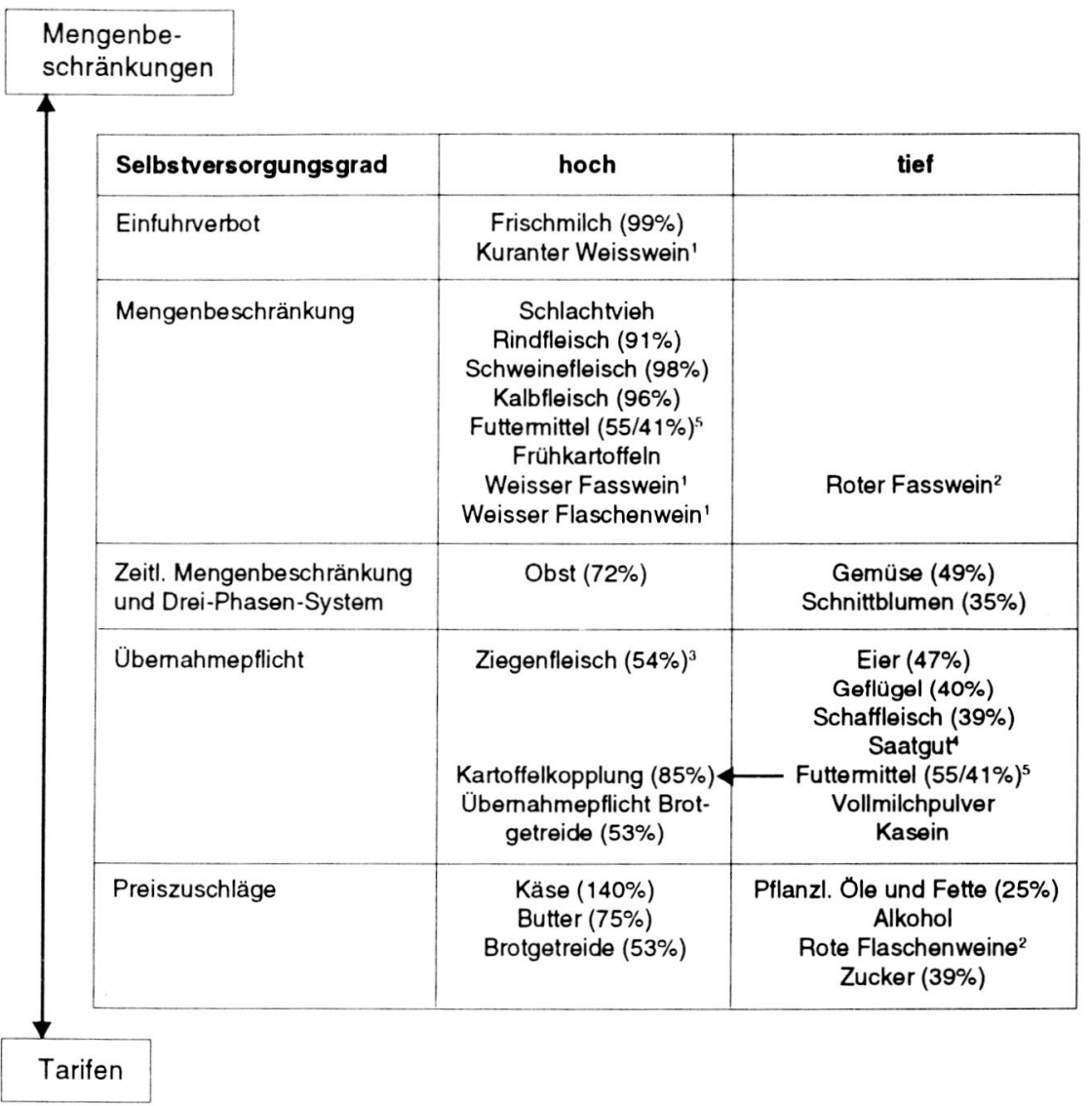

1) Weisswein: $91 \%$

2) Rotwein: $28 \%$

3) GSF 1987

4) Brotgetreide ca. $80 \%$, Futtergetreide ca. $20 \%$, Kartoffeln ca. $100 \%$

5) Ergänzungsfutter (Umsetzbare Energie/Verd. Rohprotein): gemessen an der ganzen Futterproduktion: 85/79\%

Quelle: Schweizerisches Bauernsekretariat, 1991 
lungen ein Minimaleinkommen garantiert. Eine klare Trennung der zwei Einkommenskomponenten, eine marktabhängige Komponente und Direktzahlungen würde auch zu einer neuen Dynamik in der Landwirtschaft führen. Die unternehmerischen Fähigkeiten der Bauern könnten dadurch wieder ausgeschöpft werden.

\section{d) Das Strukturproblem}

Unter dem Begriff Agrarstruktur werden Fragen nach Betriebsgrösse, Anzahl und Art der Betriebe, aber auch Aspekte der Besiedlung im Berggebiet zusammengefasst. Die Zahl der schweizerischen Landwirtschaftsbetriebe ist von rund 162'000 im Jahre 1965 auf 108'000 im Jahr 1990 gesunken. Unter der Annahme, dass die Schweizer Wirtschaft in den nächsten 20 Jahren weiterhin qualitativ hochstehende Produkte für den Inland- und vor allem für den Exportmarkt produziert, wird ein starker Abwanderungssog bestehen bleiben. Der eingeleitete Strukturwandel dürtte sich also auch in Zukunft fortsetzen. Die Zahl der Haupterwerbsbetriebe wird also weiter abnehmen, jedoch wird jeder Betrieb eine grössere Fläche bewirtschaften.

\subsection{Lösungsansätze für die Schweiz}

Die Oberziele der Landwirtschaft - Produktion von Nahrungsmitteln guter Qualität, Vorsorge für Zeiten mit gestörter Zufuhr, Landschaftsschutz und Erhaltung der dezentralen Besiedlung - sind auch heute unumstritten. Einzig die Wege, die zu diesen Zielen führen, sollen geändert werden. So soll einerseits der unternehmerischen Komponente in der Landwirtschaft vermehrt Rechnung getragen werden, anderseits werden ökologisch verträgliche Produktionssysteme angestrebt.

Wie beschrieben, weist die schweizerische Landwirtschaft hausgemachte Probleme auf, welche in konfliktträchtigen Beziehungen zueinander stehen. So ist auch der Wille zu einer Akzentverschiebung in der schweizerischen Agrarpolitik unabhängig vom Ausgang der GATT-Verhandlungen spürbar. Mit einem Paket von Selbsthilfemassnahmen und der Zustimmung zu Direktzahlungen wurde die Bereitschaft zu einer Öffnung und zu einem Strukturwandel im Interesse der überlebensfähigen Betriebe signalisiert. Mit dem Reformpaket soll eine klarere Trennung zwischen der Preis- und Einkommenspolitik erreicht werden. Der Marktpreis soll wieder vermehrt seine regulierende Funktion übernehmen, wobei das Preisgefälle zwischen der Schweiz und den europäischen Ländern verkleinert und die Dynamik der Bauern als Unternehmer gefördert werden soll. Auch aus diesen Gründen wird längerfristig eine Lockerung des Grenzschutzes angestrebt.

Die aufgezeigten Lösungsansätze sind in der Schweiz dank dem politischen Willen, die Landwirtschaft zu erhalten und zu stützen, möglich. Der politische Wille allein genügt allerdings nicht, gleichzeitig müssen die benötigten finanziellen Mittel bereitgestellt werden können. 


\section{Mögliche Auswirkungen einer Liberalisierung im Agrarhandel auf die Dritte Welt}

\subsection{Zusammenhang zwischen Agrar- und Gesamtwirtschaftspolitik}

In vielen Entwicklungsländern wird die Landwirtschaft gegenüber den andern Wirtschaftssektoren benachteiligt. Dies geschieht meistens mittels einer Kombination von überbewerteten Wechselkursen und Zoll- und Kontingentsschutz für die Industrie. Im Rahmen einer breit angelegten Studie konnte gezeigt werden, dass in vielen Entwicklungsländern die Instrumente der Gesamtwirtschaftspolitik für die Anreize in der Landwirtschaft ebenso bedeutend sind wie die Instrumente der direkten Agrarpolitik (Herrmann, 1992). Seit dem Ausbruch der Schuldenkrise 1982 versucht die Weltbank die Volkswirtschaften verschuldeter Entwicklungsländer mit Strukturanpassungsprogrammen zu sanieren. Die Korrekturen betreffen hauptsächlich die Gesamtwirtschaftspolitik, wie Finanz-, Budget-, Wechselkursund Aussenhandelspolitik und zielen auf eine Liberalisierung der Gesamtwirtschaftspolitik hin, von welcher auch positive Impulse für die Landwirtschaft erwartet werden.

\subsection{Nicht-marktwirksame Leistungen der Landwirtschaft}

In den meisten Entwicklungsländern hat die Landwirtschaft neben der Produktion auch eine Reservoirstellung inne. Die Bereitstellung neuer Arbeitsplätze kann oftmals nicht Schritt halten mit der schnell wachsenden aktiven Bevölkerung. Deshalb sind in Entwicklungsländern die sozialen Funktionen der Landwirtschaft von grösster Bedeutung. Ein Grossteil der ländlichen Bevölkerung besteht aus Familien, welche über eine grosse Zahl von Arbeitskräften, aber zuwenig Beschäftigungsmöglichkeiten verfügen. Dadurch nimmt sowohl die Migration in die Städte auf der Suche nach Arbeit wie auch die Unterbeschäftigung in der Landwirtschaft zu. Die Landwirtschaft vermag einen Teil derjenigen arbeitsfähigen Bevölkerung zu beschäftigen, welche im ausserlandwirtschaftlichen Bereich keine Arbeit findet. Zwar werden geringe Arbeitsproduktivität und Unterbeschäftigung in der Landwirtschaft beklagt, doch unter diesen Umständen muss die Unterbeschäftigung nicht als Ineffizienz, sondern als sozial integrierte Arbeitslosigkeit betrachtet werden.

Die soziale Aufgabe der Landwirtschaft kann in diesen Ländern aber nur erfültt werden, wenn die inländische Produktion, zumindest auf den Inlandmärkten zu Preisen abgesetzt werden kann, bei denen die sich ergebenden Einkommen oberhalb der Armutsgrenze liegen. Für Entwicklungsländer ist deshalb die Frage, ob durch eine Liberalisierung der Agrarmärkte die Landwirtschaft grundsätzlich gestärkt wird, von grösster Bedeutung.

Die Wohlfahrtswirkungen des Abbaus von Handelsschranken auf die Landwirtschaft lassen sich nur dann zutreffend einschätzen, wenn man die nicht marktwirksamen Leistungen in die Betrachtung einbezieht. Es handelt sich hierbei 
hauptsächlich um die sozialen und ökologischen Leistungen und den Beitrag zur Risikovorsorge (Weinschenk, 1990). Nicht marktwirksame Leistungen der Landwirtschaft sind aber sehr schwierig einzuschätzen und von Land zu Land unterschiedlich. Die folgenden Betrachtungen konzentrieren sich auf die Funktion der Landwirtschaft als Produzentin von Nahrungsmitteln und Rohstoffen, während nicht marktwirksame Leistungen vernachlässigt werden.

\subsection{Resultate aus Modellrechnungen im Bereich Nahrungsmittel}

Zur Messung des Agrarschutzes werden verschiedene Konzepte verwendet. Das im Rahmen des GATT angewandte Instrument ist der Ansatz der Producer Subsidy Equivalents (PSE). Das PSE gibt in Prozent denjenigen Teil der landwirtschaftlichen Einkommen an, welcher aus staatlicher Unterstüzung stammt. Zur Schätzung der Auswirkungen, welche eine vollständige oder teilweise Liberalisierung auf den Weltagrarhandel hätte, wurde eine Vielzahl von Modellen ausgearbeitet. Die Mehrzahl dieser Modelle befasst sich mit der Liberalisierung des Handels von Nahrungsmitteln, welche weltweit produziert werden. Tropische Getränke und Rohstoffe werden meist nicht in die Betrachtungen einbezogen, da die Hauptexporteure die Entwicklungsländer sind. Der Handel mit diesen Produkten wird somit weniger direkt von einer Liberalisierung in den OECD-Staaten beeinflusst. Indirekt besteht der Zusammenhang in der Veränderung ihrer relativen Preise zu Nahrungsmitteln. Dadurch werden die Anreize zum Anbau von tropischen Getränken und Rohstoffen verändert.

Nahrungsmittel sind auch deshalb Gegenstand der meisten Untersuchungen, da diese die Ernährung der Weltbevölkerung und die Ernährungssicherheit bestimmen und viele Entwicklungsländer heute Importeure von Nahrungsmitteln sind. In Tabelle 3 sind die Resultate einiger der wichtigsten Modellrechnungen bei einer Liberalisierung des Agrarhandels seitens der Industrieländer zusammengefasst. Dabei werden unterschiedliche Szenarien des Protektionsabbaus dargestellt.

Zietz und Valdès (1989) kommen zum Schluss, dass bei einer einseitigen Liberalisierung der OECD-Länder geringe Preiserhöhungen auf den internationalen Märkten für Weizen und Reis zu erwarten sind. Preise für Fleisch und Zucker würden stärker ansteigen, während die Preise für Futtermittel (Soya, Grobgetreide) sogar sinken würden. Die Resultate anderer in der Tabelle aufgeführter Studien lassen auf grössere Preisänderungen infolge der Liberalisierung schliessen. Die Ergebnisse dieser partiellen Gleichgewichtsmodelle dürfen jedoch nur als Trends verstanden werden, da Interaktionen zwischen einzelnen Produkten nur teilweise erfasst werden. Auch die den Berechnungen zugrunde gelegte Basisperiode, sowie Annahmen bezüglich Preis- und Einkommenselastizitäten und Wachstumsraten der Gesamtwirtschaft, beeinflussen die Ergebnisse. Insgesamt ist aber doch anzunehmen, dass durch das Wegfallen von Überschüssen aus den Industrienationen allgemein stabilere Weltmarktpreise entstehen werden. 
Tabelle 3: Änderung der Weltmarktpreise bei einer Liberalisierung des Agrarhandels in den Industrieländern (Basis 1981-83)

\begin{tabular}{|c|c|c|c|c|c|c|}
\hline $\begin{array}{l}\text { Abbau } \\
\text { PSE/CSE }\end{array}$ & Weizen & $\begin{array}{l}\text { Grob- } \\
\text { getreide }\end{array}$ & Reis & $\begin{array}{l}\text { Rind- } \\
\text { fleisch }\end{array}$ & Zucker & Soya \\
\hline \multicolumn{7}{|l|}{ Zietz/Valdès 90: } \\
\hline $10 \%$ & 0.4 & -0.1 & 0.2 & 0.9 & 1.5 & -0.3 \\
\hline $50 \%$ & 2.9 & -0.7 & 0.9 & 4.9 & 7.6 & -1.5 \\
\hline $100 \%$ & 3.5 & -2.8 & 1.7 & 10.5 & 15.0 & -4.0 \\
\hline \multicolumn{7}{|l|}{ Andere Studien: } \\
\hline OECD-87 (10\%) & -0.1 & -0.3 & 0.1 & 1.5 & 1.0 & -1.0 \\
\hline IIASA $(100 \%)$ & 18.0 & 11.0 & 21.0 & 17.0 & - & - \\
\hline \multicolumn{7}{|l|}{ Tyers/Anderson } \\
\hline \multicolumn{7}{|l|}{$1987(100 \%)$} \\
\hline a) & 9.0 & 3.0 & 10.0 & 21.0 & 10.00 & - \\
\hline b) & 25.0 & 3.0 & 18.0 & 43.0 & 22.0 & - \\
\hline \multicolumn{7}{|l|}{ Zietz/Valdès 86: } \\
\hline c) $(100 \%)$ & 12.1 & 11.0 & - & 17.4 & 18.2 & - \\
\hline \multicolumn{7}{|c|}{$\begin{array}{l}\text { a) basiert auf Protektion } 1980-82 \\
\text { b) basiert auf vorausgeschätzter Protektion } 1988 \\
\text { c) basiert auf Protektion } 1979-81\end{array}$} \\
\hline \multicolumn{7}{|c|}{ Quelle: Zietz und Valdès, in Brown und Goldin, 1992} \\
\hline
\end{tabular}

Wie Tabelle 4 zeigt, sind die Resultate der Modellrechnungen bezüglich einer weltweiten Liberalisierung des Handels mit Nahrungsmitteln nicht einheitlich. Aufgrund steigender Anreize zur Marktproduktion für die Produzenten in den Entwicklungsländern dürfte sich das Angebot stark ausdehnen und trotz Bevölkerungs- und Wirtschaftswachstum zu sinkenden Preisen für Grundnahrungsmittel wie Getreide, Reis und Zucker führen, da die Angebotsausdehnung die Nachfragesteigerung übertreffen dürtte (Zietz und Valdès, 1990). Den Modellen wird ein positives Wirtschaftswachstum der Entwicklungsländer unterstellt. Unter dieser Annahme würde die Nachfrage nach superioren Gütern wie Fleisch in diesen Ländern zunehmen und die Preise ansteigen. 
Tabelle 4: Änderung der Weltmarktpreise bei einer weltweiten Liberalisierung des Agrarhandels (Basis 1981-83)

\begin{tabular}{|l|c|c|c|c|c|c|}
\hline $\begin{array}{l}\text { 100\% Abbau } \\
\text { PSE/CSE }\end{array}$ & Weizen & $\begin{array}{c}\text { Grob- } \\
\text { getreide }\end{array}$ & Reis & $\begin{array}{c}\text { Rind- } \\
\text { fleisch }\end{array}$ & Zucker & Soya \\
\hline $\begin{array}{l}\text { Zietz/Valdès 90: } \\
\text { El }\end{array}$ & -13.6 & -20.9 & -21.8 & 2.9 & -12.1 & -11.5 \\
IL + EL & -11.7 & -24.4 & -21.2 & 13.3 & 0.8 & -15.9 \\
IIASA: & 5.0 & 4.0 & 1.0 & -3.0 & - & - \\
$\begin{array}{l}\text { EI } \\
\text { IL + EL }\end{array}$ & 23.0 & 13.0 & 16.0 & 11.0 & - & - \\
$\begin{array}{l}\text { Tyers/Anderson } \\
\text { 1987: }\end{array}$ & 10.0 & 2.0 & -8.0 & 13.0 & -1.0 & - \\
IL + EL & & & & & & \\
\hline
\end{tabular}

Quelle: Zietz und Valdès, in Brown und Goldin, 1992

\subsection{Sind Entwicklungsländer Gewinner oder Verlierer der Liberalisierung?}

Die Frage, ob Entwicklungsländer grundsätzlich zu den Gewinnern oder Verlierern einer Liberalisierung des Agrarhandels gehören, lässt sich nicht einheitlich beantworten, denn bezüglich des Agrarhandels gibt es "die" Entwicklungsländer nicht. So muss zwischen Nahrungsmittelimporteuren und -exporteuren unterschieden werden. Zudem kann ein Nahrungsmittelimporteur gleichzeitig andere Agrargüter exportieren. In diesem Fall ist die Struktur der Agrarexporte zu berücksichtigen sowie das Potential derjenigen Produkte, für welche nach der Liberalisierung ein höherer Erlös erzielt werden kann.

Welche Auswirkungen von einer Liberalisierung zu erwarten sind, ist abhängig wer in welchem Ausmass liberalisiert. Wie beschrieben, sind die Industrieländer gezwungen, auch ihre Agrarbudgets zu redimensionieren. Somit ist der Wille zum Abbau von handelshemmenden Massnahmen vorhanden. Doch noch ist nicht entschieden, ob die Protektion des Agrarhandels in den OECD-Staaten ganz oder nur teilweise abgebaut wird. Auch ist noch nicht klar, wie die Länder der Dritten Welt mit der von ihnen im Agrarsektor praktizierten Diskriminierung durch Preis-, Fiskalund Wechselkurspolitik verfahren werden.

Um die Frage der Auswirkungen einer Handelsliberalisierung in Entwicklungsländern schlüssig beantworten zu können, müssen auch die Entwicklung der Gesamtwirtschaft der einzelnen Länder, die Stellung der Landwirtschaft und ihre nicht-marktwirksamen Leistungen mitberücksichtigt werden. Die Ergebnisse der Modellrechnungen zeigen, dass für kaum ein landwirtschaftliches Produkt längerfristig steigende Weltmarktpreise zu erwarten sind. 
Nahrungsmittel tragen heute etwa einen Drittel zu den Erlösen der landwirtschaftlichen Exporte der Entwicklungsländer bei. Brown und Goldin (1992) kommen zum Schluss, dass bei einer einseitigen Liberalisierung der OECD-Staaten, diese die Hauptgewinner wären, da die hohen Kosten des Protektionismus abgebaut werden. Indirekte Gewinne würde auch das weltweite Handelssystem dank höherer Transparenz und Preisstabilität erfahren. Aber auch die Entwicklungsländer würden von der Liberalisierung profitieren und ihre Nahrungsmittelproduktion ausdehnen. Dadurch könnte der Selbstversorgungsgrad erhöht und die Ernährungssicherheit gesteigert werden. Verlierer wären hauptsächlich diejenigen Entwicklungsländer, welche auf Nahrungsmitteleinfuhren angewiesen sind.

Konsumenten in Entwicklungsländern müssten bei einer Liberalisierung mit höheren Preisen für Nahrungsmittel rechnen. Hier ist entscheidend, ob durch geeignete Massnahmen der Gesamtwirtschaftspolitik das Wirtschaftswachstum gefördert und neue Arbeitsplätze geschaffen werden können. Heute werden in vielen Entwicklungsländern die Preise für Nahrungsmittel vom Staat festgelegt und kontrolliert. Diese liegen oftmals unter den Weltmarktpreisen und bringen so grosse Vorteile für die Konsumenten, gleichzeitig aber entscheidende Nachteile für die Produzenten, da die tiefen Preise an die inländische Landwirtschaft weitergeleitet werden. Durch die Liberalisierung würden die Subventionen zugunsten der Konsumenten und zulasten der Landwirtschaft aufgehoben. Für benachteiligte Bevölkerungsschichten mit geringen Einkommen müssten jedoch Programme der Abgabe verbilligter Nahrungsmittel ausgearbeitet werden. Die Kosten solcher Programme, welche eine gezielte Unterstützung armer Bevölkerungsschichten beinhalten, dürtten die Kosten der heutigen allgemeinen Konsumsubventionen nicht überschreiten. Die Durchführung dieser Programme könnte in Ländern, welchen die nötigen Mittel zur Finanzierung gezielter Massnahmen fehlen, durch die internationale Gemeinschaft gefördert werden.

Der Handel mit Rohstoffen und tropischen Getränken ist geprägt durch hohe Ausfuhrzölle in den Produzentenländern und abgestufte Einfuhrzölle je nach Verarbeitungsgrad in den Importländern. Bei einer Liberalisierung des Handels seitens der Importländer würden sich die Einnahmen für die Exporteure erhöhen. Bei einer Liberalisierung auch in den Erzeugerländern, würden die Produzenten nach der Aufhebung der Exportsteuern und allfälligen Wechselkursverzerrungen vorerst höhere Preise erzielen und dadurch das Angebot ausdehnen. In den Hauptabnahmeländern dürfte der Konsum von Rohstoffen und tropischen Getränken jedoch kaum ansteigen, die Nachfrage ist gesättigt. Einem steigenden Angebot würde somit eine konstante Nachfrage gegenüberstehen, wodurch die Weltmarktpreise längerfristig wieder sinken würden. Durch die Aufhebung der Importbesteuerung in den OECD-Ländern könnten die Produzentenländer jedoch ihren Anteil an verarbeiteten Produkten beträchtlich steigern, da die komparativen Kostenvorteile der Verarbeitung ausgenutzt werden könnten.

Infolge der Verschiebung der relativen Preise zugunsten der Nahrungsmittel, dürfte sich die Produktion von Rohstoffen zugunsten derjenigen von Nahrungsmitteln verlagern. Bei Änderungen der Produktionsausrichtung sind die Agrarpolitik und die Agrarstruktur von entscheidender Bedeutung. In vielen Entwicklungs- 
ländern besteht ein grosser Gegensatz zwischen Gross- und Kleinbetrieben. Während die Kleinbetriebe in erster Linie Nahrungsmittel produzieren, übernehmen Grossbetriebe und Plantagen die Erzeugung von landwirtschaftlichen Rohstoffen. Subsistenzwirtschaft und die Nahrungsmittelproduktion wird oftmals vor allem in Lateinamerika - in Hanglagen verdrängt, während das fruchtbarere Land im Talgebiet durch extensive Viehhaltung genutzt wird. Auch hier wird ein Umdenken nötig sein, damit der Boden sowohl agronomisch als auch ökologisch sinnvoll genutzt wird.

Die zukünftige Politik der osteuropäischen Länder wird den internationalen Handel im Agrarbereich beeinflussen. Der Konsum von Zitrusfrüchten, Bananen, Kaffee und andern tropischen Produkten ist in diesen Ländern sehr gering. Durch die beschränkte Verfügbarkeit dieser Produkte war der Konsum eingeschränkt; bis heute ist die Nachfrage, bedingt durch die gesunkene Kaufkraft der Konsumenten, noch nicht stark gestiegen. Das zukünftige Wirtschaftswachstum wird die Einkommen der Konsumenten und damit die Nachfrage nach tropischen Produkten bestimmen. Für Entwicklungsländer, hauptsächliche Exporteure tropischer Produkte, könnte sich hier bei günstiger wirtschaftlicher Entwicklung des ehemaligen Ostblockes ein wichtiger neuer Markt entwickeln.

Seit den 70er Jahren war die ehemalige UdSSR auch wichtiger Importeur von Getreide, Fleisch und Milchprodukten, wobei die UdSSR oftmals auf dem Weltmarkt nicht absetzbare Überschüsse zu Vorzugspreisen übernahm. Ohne diese Übernahme wären die Weltmarktpreise wahrscheinlich noch tiefer gefallen. Wie sich die osteuropäischen Länder in Zukunft entwickeln werden, ist noch ungewiss. Unbestritten bleibt, dass in den osteuropäischen Ländern ein grosses Produktionspotential für landwirtschaftliche Güter ungenügend genutzt wird. Dies gilt hauptsächlich für Nahrungsmittel, welche weltweit angebaut werden wie Getreide und Produkte aus der Viehhaltung. Gemäss der geographischen Lage dieser Länder beschränkt sich der Anbau von nicht kälteresistenten Gemüsen und Früchten in Freilandkulturen auf die südlichen Länder wie Bulgarien, Rumänien, Ungarn etc. Wie sich die osteuropäischen Länder in Zukunft verhalten werden und wie sich ihre Landwirtschaft entwickelt, ist somit von grösster Bedeutung für den internationalen Handel mit Agrarprodukten.

\section{Folgerungen für den Handel mit Agrarprodukten der Schweiz mit Entwicklungsländern}

In Punkt 3 wurde gezeigt, wie sich die Schweiz durch Massnahmen der Aussenhandelspolitik vor Importen aus dem Ausland schützt. Im folgenden soll nun abgeschätzt werden, ob die Entwicklungsländer durch die Liberalisierung des Agrarhandels mit erweiterten Exportmöglichkeiten in die Schweiz rechnen können und welche Konsequenzen für die Schweiz zu erwarten sind.

Die Schweiz importierte 1990 für 8.1 Milliarden Franken land- und forstwirtschaftliche Produkte, das sind $8.4 \%$ der Gesamtimporte. Rund $75 \%$ dieser land- 
und forstwirtschaftlichen Produkte stammten aus dem europäischen Raum, allein die EG lieferte $67 \%$ der Importe, weitere $8 \%$ stammen aus den EFTA-Ländern (Oberzolldirektion, 1991). Die Ausfuhren land- und forstwirtschaftlicher Produkte der Schweiz beliefen sich 1990 auf 0.3 Milliarden Franken, davon wurden $70 \%$ in den europäischen Raum exportiert. Somit kann festgehalten werden, dass der Austausch landwirtschaftlicher Produkte zwischen der Schweiz und den Entwicklungsländern von untergeordneter Bedeutung ist.

Die liberalsten Einfuhrbestimmungen bestehen für Produkte, welche in der Schweiz nicht angebaut werden und wofür es keine inländischen Substitute gibt. Es sind dies hauptsächlich tropische Getränke und gewisse landwirtschaftliche Rohstoffe (z.B. Baumwolle). Die Einfuhr dieser Produkte ist frei, es werden aber Fiskalzölle erhoben. Die schweizerischen Einfuhren an Grünkaffee lagen 1990 bei $65^{\prime} 000$ Tonnen, das sind $2.7 \%$ der europäischen Kaffee-Einfuhren. 52\% der schweizerischen Kaffeeimporte wurden von den vier lateinamerikanischen Ländern Brasilien, Kolumbien, Costa Rica und Guatemala geliefert (Oberzolldirektion). Ähnliche Proportionen ergeben sich für die andern tropischen Getränke und für Rohstoffe. Da der schweizerische Markt für diese Produkte gesättigt ist, dürften sich die Importmengen, auch nach dem Abbau aller Handelsschranken, kaum stark verändern.

Auch die Einfuhr von Zucker ist mengenmässig nicht beschränkt, doch muss eine Importbewilligung eingeholt werden. In den 60er Jahren lieferten Kuba, Mexiko, die Dominikanische Republik und andere Entwicklungsländer rund 30\% des eingeführten Zuckers zu Weltmarktpreisen. Im Laufe der folgenden 20 Jahre schieden zuerst die Entwicklungsländer Süd- und Mittelamerikas als Zuckerlieferanten für die Schweiz aus, dann Osteuropa. 1989 stammten 99.8\% des eingeführten Zuckers aus der EG (Rieder, Egger, 1992). Die Produktionskosten des europäischen Rübenzuckers sich jedoch weit höher als jene von Rohrzucker aus den Entwicklungsländern. Da aber der Export von EG-Zucker subventioniert wird, können komparative Kostenvorteile der Entwicklungsländer nicht zum Tragen kommen. Wie die Entwicklung der Zuckerimporte zeigt, profitiert die Schweiz von den Exportsubventionen der EG. Bei einer Handelsliberalisierung müssten diese Subventionen aufgehoben werden und die Entwicklungsländer könnten wieder vermehrt ihre komparativen Kostenvorteile nutzen. Dadurch würden die Zuckerimporte der Schweiz aus Entwicklungsländern wieder ansteigen.

Der Selbstversorgungsgrad bei Rindfleisch betrug im Durchschnitt der letzten 10 Jahre 95\%. Dadurch erklärt sich, dass der Import von Rindfleisch mengenmässig beschränkt ist. Die Kontingente werden je nach Marktsituation (Produzentenpreise im Inland) festgelegt. Die Herkunftsländer der Rindfleischimporte sind hauptsächlich Argentinien und Brasilien, wobei zu zwei Dritteln Nierstücke und Bindenstotzen geliefert werden. Infolge des Fleischpreisgefälles zwischen der Schweiz und dem Ausland würden bei einer Aufhebung des Aussenhandelsschutzes die Fleischpreise im Inland stark sinken und die Produktion abnehmen. $\mathrm{Da}$ der Konsum von Rindfleisch sowohl vom Einkommen als auch vom Preis abhängig ist, dürfte sich die Nachfrage bei Wirtschaftswachstum und tiefen Preisen ausdehnen. Von dieser Situation könnten einerseits die schweizerischen Kon- 
sumenten, anderseits exportorientierte Entwicklungsländer mit tiefen Produktionskosten wie Argentinien und Brasilien profitieren.

Obst und Gemüse, welches auch im Inland produziert werden kann, ist dem Drei-Phasen-System unterstellt (siehe Abschnitt 4.1). Rund $85 \%$ der traditionellen Obstimporte und $\mathbf{5 0} \%$ der Südfrüchte stammen aus den EG-Ländern Italien, Frankreich und Spanien. Entwicklungsländer sind an den schweizerischen Importen mit "Contre-Saison"-Produkten und Südfrüchten beteiligt, welche frei importiert werden können, da sie die inländische Produktion nicht direkt konkurrieren. Allerdings bestehen oft Substitutionsbeziehungen zwischen liberalisiertem Importobst (z.B. Pfirsiche) und zu schützenden einheimischen Saisonfrüchten (z.B. Zwetschgen). Bei einer Liberalisierung müsste das Drei-Phasen-System, welches den Schweizer Markt bei hohem Inlandangebot gegenüber den Importen schützt, aufgehoben werden. Dadurch würde die inländische Produktion direkt durch das Ausland konkurriert, was Preissenkungen und Produktionsabnahme im Inland zur Folge hätte. Die südlichen EG-Länder würden ihre Exporte in die Schweiz steigern und die schweizerischen Konsumenten könnten Obst und Gemüse günstiger einkaufen. Aber auch geographisch günstig gelegene Entwicklungsländer wie z.B. Länder des nördlichen Afrikas könnten ihre Exporte ausdehnen. Die Konkurrenz würde hauptsächlich zwischen Ländern mit ähnlichen Produktionsbedingungen und Distanzen zum schweizerischen Markt wie der südliche EG-Raum, der Nahe Osten (Israel) und das nördliche Afrika (Tunesien, Marokko, Algerien) zunehmen.

Zusammenfassend kann festgehalten werden, dass durch eine Liberalisierung des Agrarhandels und Abbaus aller Handelshemmnisse, die Produzentenpreise in der Schweiz sinken werden. In dieser Situation kann die schweizerische Landwirtschaft nur dann überleben und ihre gesetzlich verankerten Leistungen erbringen, wenn die Einkommensausfälle durch Direktzahlungen ausgeglichen werden (siehe Abschnitt 4). Hauptprofiteure wären die inländischen Konsumenten, welchen günstigere Nahrungsmittel zur Verfügung stehen würden. Aber auch Entwicklungsländer könnten ihre Exporte landwirtschaftlicher Produkte - infolge komparativer Kostenvorteile - erhöhen. Der schweizerische Agrarmarkt ist aber nur sehr klein und für den internationalen Agrarhandel nicht von Bedeutung. Für Entwicklungsländer wird vielmehr entscheidend sein, wie sich der EG-Markt entwickelt.

\section{Literatur}

Brown M., Goldin I., The Future of Agriculture: Developing Country Implications, OECD, Paris 1992

Egger U., Rieder P., Clemenz D., Internationale Agrarmärkte, Verlag der Fachvereine Zürich, Zürich 1992

Eidgenössische Oberzolldirektion, Schweizerische Aussenhandelsstatistik, Bern 1992

Herrmann R., Friedrich L., Wirtschaftliche Liberalisierung in Industrie- und Entwicklungsländern, Verlag Weltarchiv GMBH, Hamburg 1992 
Rieder P., Egger U., Flückiger S., Schweizerische Agrarmärkte, Verlag der Fachvereine Zürich, Zürich 1992

Rieder P., Die Landwirtschaft unter Druck, in: Der Monat in Wirtschaft und Finanz, Schweizerischer Bankverein, Juni 1991

Schweizerisches Bauernsekretariat, Statistische Erhebungen und Schätzungen, Brugg 1991

Weinschenk G., Wieviel Freihandel enträgt die Landwirtschaft?, Universität Hohenheim, unveröffentlicht, Stuttgart, 1991 